\title{
Validation of self-reported figural drawing scales against anthropometric measurements in adults
}

Julia Dratva ${ }^{1,2, *}$, Randi Bertelsen ${ }^{3}$, Christer Janson ${ }^{4}$, Ane Johannessen ${ }^{3,5}$, Bryndis Benediktsdóttir ${ }^{6}$, Lennart Bråbäck ${ }^{7}$, Shyamali C Dharmage ${ }^{8}$, Bertil Forsberg ${ }^{7}$, Thorarinn Gislason ${ }^{6}$, Debbie Jarvis ${ }^{9}$, Rain Jogi ${ }^{10,11}$, Eva Lindberg ${ }^{4}$, Dan Norback ${ }^{4}$, Ernst Omenaas ${ }^{5}$, Trude D Skorge ${ }^{3}$, Torben Sigsgaard ${ }^{12}$, Kiell Toren ${ }^{13}$, Marie Waatevik ${ }^{5}$, Gundula Wieslander $^{4}$, Vivi Schlünssen ${ }^{12}$, Cecilie Svanes $3,14, \uparrow$ and Francisco Gomez Real ${ }^{5,15,} \uparrow$ ${ }^{1}$ Department of Epidemiology and Public Health, Swiss Tropical and Public Health Institute, Socinstrasse 57, PO Box 4002, Basel, Switzerland: ${ }^{2}$ University of Basel, Basel, Switzerland: ${ }^{3}$ Department of Occupational Medicine, Haukeland University Hospital, Bergen, Norway: ${ }^{4}$ Department of Medical Sciences, Uppsala University, Uppsala, Sweden: ${ }^{5}$ Department of Clinical Sciences, University of Bergen, Bergen, Norway: ${ }^{6}$ Department of Allergy, Respiratory Medicine and Sleep, Landspitali University Hospital, Reykjavik, Iceland: ${ }^{7}$ Occupational and Environmental Medicine, Department of Public Health and Clinical Medicine, Umeå University, Umeå, Sweden: ${ }^{8}$ Allergy and Lung Health Unit, Centre for Epidemiology and Biostatistics, Melbourne School of Population and Global Health, University of Melbourne, Melbourne, Australia: ${ }^{9}$ Faculty of Medicine, National Heart \& Lung Institute, Imperial College, London, UK: ${ }^{10}$ Lung Clinic, Foundation Tartu University Clinics, Tartu, Estonia: ${ }^{1}$ Department of Pulmonary Medicine, Tartu University, Tartu, Estonia: ${ }^{12}$ Department of Public Health, Aarhus University, Aarhus, Denmark: ${ }^{13}$ Occupational and Environmental Medicine, Sahlgrenska Academy, University of Gothenburg, Gothenburg, Sweden: ${ }^{14}$ Centre for International Health, University of Bergen, Bergen, Norway: ${ }^{15}$ Department of Gynecology and Obstetrics, Haukeland University Hospital, Bergen, Norway

Submitted 25 June 2015: Final revision received 15 December 2015: Accepted 11 January 2016: First published online 16 February 2016

\begin{abstract}
Objective: The aim of the present study was to validate figural drawing scales depicting extremely lean to extremely obese subjects to obtain proxies for BMI and waist circumference in postal surveys.

Design: Reported figural scales and anthropometric data from a large populationbased postal survey were validated with measured anthropometric data from the same individuals by means of receiver-operating characteristic curves and a BMI prediction model.

Setting: Adult participants in a Scandinavian cohort study first recruited in 1990 and followed up twice since.

Subjects: Individuals aged 38-66 years with complete data for BMI ( $n$ 1580) and waist circumference ( $n$ 1017).

Results: Median BMI and waist circumference increased exponentially with increasing figural scales. Receiver-operating characteristic curve analyses showed a high predictive ability to identify individuals with BMI $>25.0 \mathrm{~kg} / \mathrm{m}^{2}$ in both sexes. The optimal figural scales for identifying overweight or obese individuals with a correct detection rate were 4 and 5 in women, and 5 and 6 in men, respectively. The prediction model explained $74 \%$ of the variance among women and $62 \%$ among men. Predicted BMI differed only marginally from objectively measured BMI.

Conclusions: Figural drawing scales explained a large part of the anthropometric variance in this population and showed a high predictive ability for identifying overweight/obese subjects. These figural scales can be used with confidence as proxies of BMI and waist circumference in settings where objective measures are not feasible.
\end{abstract}

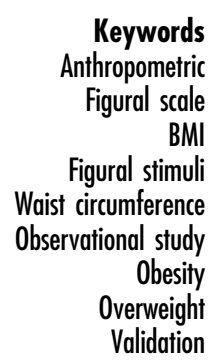

The obesity epidemic has drawn attention to the role of metabolic factors in the aetiology of non-communicable diseases. Considerable evidence points to the adverse impact of obesity on cardiometabolic and respiratory

$\dagger \mathrm{C}$ Svanes and FG Real share last authorship.

diseases $^{(1,2)}$. Obesity's adverse effect may be related to metabolic and inflammatory pathways ${ }^{(3,4)}$ and/or actual weight of body fat ${ }^{(5,6)}$.

In clinical and epidemiological studies, BMI or waist-to-hip ratio is most commonly used to define anthropometric status. These proxies of body fat are related to clinical health 
outcomes $^{(1,7)}$. More sophisticated measures of body composition, such as bioimpedance measurements, are also used to assess body fat and fat-free mass ${ }^{(8)}$. However, in large population-based studies or studies in remote settings, neither option is feasible. Figural stimuli, representing a range of figural drawing scales (figural scales) from extremely lean to extremely obese, are an easy-to-administer self-reported measure of body image. First introduced and validated by Stunkard et al. in $1983^{(9)}$, figural scales have been used in many studies in place of measured or self-reported height and weight ${ }^{(9-12)}$ or to assess body satisfaction by comparing an individual's perception of his/ her body with his/her ideal body image ${ }^{(13,14)}$.

The latest Respiratory Health in Northern Europe (RHINE) survey, performed in 2010-2012, introduced modernised figural stimuli for men and women, with nine categories, similar to Stunkard's figural scales. The purpose of introducing figural stimuli in the survey was twofold. First, the figural scales complemented self-reported current height and weight by adding information on body fat distribution. Second, if the figural scales proved to be a valid instrument in the present, they could be used to assess anthropometric status at specific time points in the past (time of menopause, 55 years, 40 years, 30 years) and thereby provide a history of anthropometrics, often missing in epidemiological research. Figural scales could also be a valid alternative to or an additional instrument for assessing anthropometric data in cultural settings in which people do not know their height and weight because they are not commonly measured or because anthropometric measurements cannot be acquired.

In the current study we validated the reported current figural scales with measured current BMI in a sub-sample of RHINE participants with data on measured height, weight and waist circumference (WC). Our aim was to investigate the predictive power of figural scales to identify individuals at metabolic risk.

\section{Materials and methods}

The RHINE study population (www.rhine.nu) consists of the population-based study sample recruited for the first stage of the European Community Respiratory Health Survey (ECRHS, 1990-1994; www.ecrhs.org) ${ }^{(15)}$. Men and women aged 20-44 years were randomly selected from population registers within specific boundaries and were sent a questionnaire by post ( $n 21$ 802; response rate 83.7\%). RHINE study centres are located in Reykjavik, Iceland; Bergen, Norway; Umeå, Uppsala and Gothenburg, Sweden; Aarhus, Denmark; and Tartu, Estonia. The study was approved by the local ethics commissions of each study centre. All participants provided written informed consent. Since the initial survey, participants have been followed up twice by postal questionnaire ${ }^{(16)}$. The analyses presented here were performed on a sub-sample of RHINE III participants who also participated in the third ECRHS clinical study
(2011-2012) and had complete data on objectively measured BMI and current figural scales ( $n$ 1580; see online supplementary material 1, Supplemental Fig. 1).

\section{Participants' data}

Through the postal questionnaires, RHINE III collected data on current and past health, lifestyle and socioeconomic status, including self-reported height and weight. BMI was defined as weight/height ${ }^{2}$. Education was defined based on the highest educational degree achieved (obligatory, secondary or tertiary levels).

\section{Figural scales}

The figural stimuli introduced during RHINE III were designed specifically for the survey by Alejandro Villén-Real (Fig. 1) and based on Stunkard's body image scales $^{(9)}$. Participants were asked to tick the figural scale that best described their current figure.

\section{Anthropometric measures}

Body weight, height and waist were measured following the ECRHS standard protocol and using calibrated scales and tape bands at the RHINE study centres. Obese (BMI $>30 \cdot 0$ $\left.\mathrm{kg} / \mathrm{m}^{2}\right)$ and overweight (BMI $=25 \cdot 0-30 \cdot 0 \mathrm{~kg} / \mathrm{m}^{2}$ ) participants, as well as those with a WC of $>88 \mathrm{~cm}$ in women and $>102 \mathrm{~cm}$ in men, were considered to be 'at risk',(17).

\section{Statistical methods}

Study participants' characteristics were stratified by sex. We compared the prevalence of characteristics among participants with and without objective anthropometric data, as well as between participants who had answered the question on body image and those who had not. In a multivariable logistic regression, we investigated the odds of non-response to the figural scales (see online supplementary material 1 , Supplemental Table 1 ).

The median and interquartile range of objectively measured BMI (oBMI), waist circumference (WC) and self-reported BMI (sBMI) were calculated for each body shape. Differences between oBMI and sBMI were assessed. Spearman correlations between the anthropometric measures and figural scales were calculated. Receiveroperating characteristic (ROC) curves $^{(18)}$ were computed to investigate the ability of the figural stimuli to identify subjects 'at risk' as defined above (obese (BMI $>30 \cdot 0 \mathrm{~kg} / \mathrm{m}^{2}$ ) $v$. non-obese; overweight (BMI $\left.>25.0 \mathrm{~kg} / \mathrm{m}^{2}\right) \quad v$. nonoverweight; females and males with $\mathrm{WC}>88 \mathrm{~cm}$ and $>102 \mathrm{~cm}$, respectively, $v$. females and males with WC below these values). ROC curves plot the true positive rate (correct detection) against the false positive rate (false alarm) for each figural scale. The area under the curve (AUC) is a measure of test accuracy ${ }^{(19)}$. ROC curves also visually facilitate identification of the best possible figural scale (optimal sensitivity and specificity criterion), which allows discrimination between obese and non-obese subjects with 
(a)

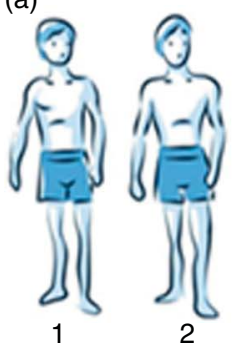

(b)
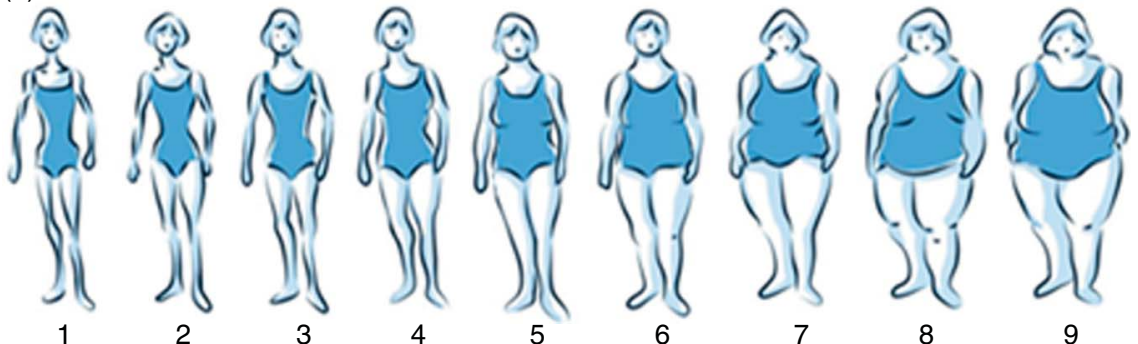

Fig. 1 Figural scales for (a) men and (b) women introduced in the Respiratory Health in Northern Europe (RHINE III) survey

maximum sensitivity (correct detection) and the least loss of specificity (correct rejection).

ROC curve analyses were performed separately for different sex and age groups ( $<52$ years $v . \geq 52$ years, mean age). The empirical optimal sensitivity and specificity criterion and the Youden index were calculated for figural scales.

After dividing the study population into two random data sets, a BMI prediction model was developed with one of the samples and validated in the other. In a step-wise backward modelling procedure, the number of covariates was reduced, based on a significance level of $0 \cdot 2$, to a final model with the highest explanatory power (adjusted $r^{2}$; Akaike criterion ${ }^{(20)}$ ). Models were built for men and women separately. In the model, oBMI was logtransformed (log BMI) to account for oBMI's left-skewed distribution. The predictive model for $\log$ BMI in women included: figural scales corresponding to current figure, age, educational status, current use of hormonal replacement therapy (HRT), prevalence of menopausal symptoms ever (MP symptoms ever) and chronic disease status (i.e. asthma and diabetes), as well as study centre as a random variable. The male predictive model included: current figural scales, age, educational status, smoking status, CVD status and study centre. The coefficients of the predictive model were then applied to the second random sample to predict log BMI. The difference between predicted log BMI and measured log BMI was calculated and the values were converted back into the original units (using $\exp (x)$ ).

\section{Results}

Of the RHINE III survey population ( $n$ 12 660), 93\% filled in current figural scales. Of these respondents, those participating in the ECRHS clinical assessment (and therefore with measured oBMI and WC) differed significantly from those without objective anthropometric measures. Differences were found with respect to age ( 52.6 years $v .51 .5$ years) and smoking status (15\% v. $17 \%)$. Participants with objective measures also had a higher prevalence of respiratory disease $(27 \% v .12 \%)$, since about a third of the random ECRHS sample had been recruited based on symptoms suggestive of asthma ${ }^{(15)}$. However, metabolically important measures such as physical exercise or diabetes were not significantly different between the two groups (see online supplementary material 1, Supplemental Table 1). The multivariable logistic regression yielded a slightly higher odds of non-responders among older participants $(\mathrm{OR}=1.03$ per year of age, $95 \%$ CI 1.02 , 1.04; see online supplementary material 1, Supplemental Table 2) and participants with high educational status $(\mathrm{OR}=0.81,95 \% \mathrm{CI} 0.73,0.91)$, and a somewhat lower ratio among women $(\mathrm{OR}=0.74,95 \%$ CI $0.64,0 \cdot 87$; Supplemental Table 2).

The analytic sample included slightly more women (58\%) than men ( $42 \%)$. The mean age of the overall sample was 52.4 years (SD 7.1), ranging from 38 to 66 years. With respect to anthropometric measures, the mean oBMI was $26.5 \mathrm{~kg} / \mathrm{m}^{2}$ (women, 25.7 (sD 6.2 ) kg/m² men, $26.9(\mathrm{sD} 4.7) \mathrm{kg} / \mathrm{m}^{2}$ ) and the mean WC was $94.3 \mathrm{~cm}$ (women, 87.8 (SD 17.6) cm; men, $98 \cdot 6$ (SD 15.1) cm).

With increasing figural scale, from scale 1 (extremely lean) to scale 9 (extremely obese), the median, 25th and 75th percentiles of oBMI and WC increased (Fig. 2). The self-reported BMI percentiles showed a comparative increase (see online supplementary material 1, Supplemental Table 3). The overall Spearman correlation coefficient between oBMI and sBMI was very high (0.94), with a difference between sexes (women, 0.944; men, 0.928; both significant $P<0 \cdot 001$, sex difference $P=0 \cdot 02$ ). 
(a)

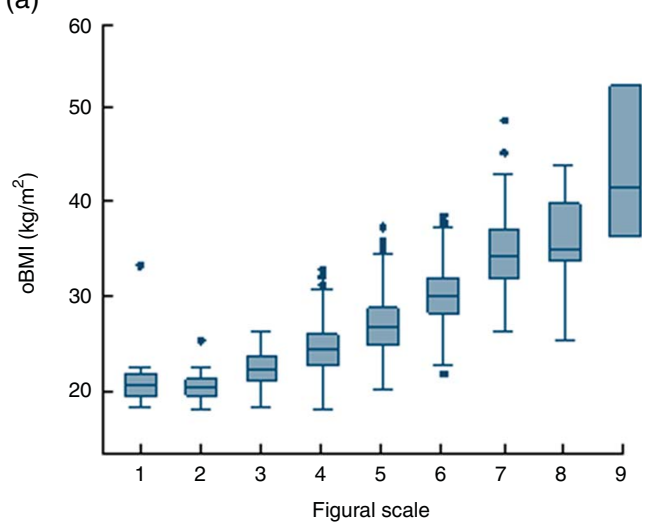

(c)

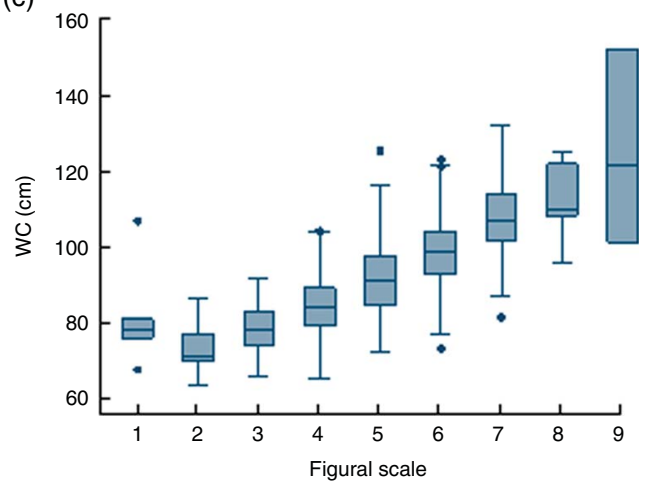

(b)

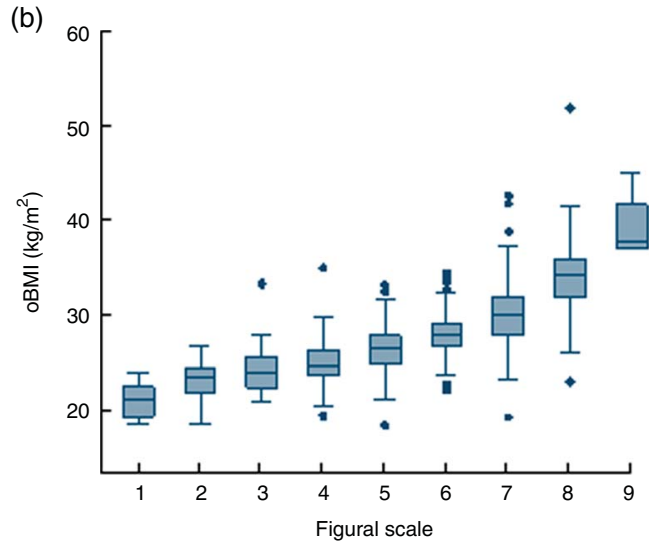

(d)

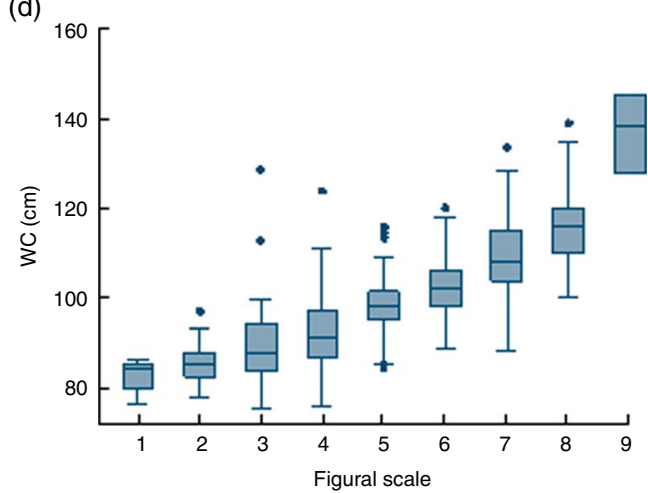

Fig. 2 Box-and-whisker plots showing the distribution of oBMI and WC by figural scale, according to sex, in Scandinavian adults aged 3866 years (sub-sample of the RHINE III): (a) oBMI in women ( $n$ 674); (b) oBMI in men ( $n$ 769); (c) WC in women ( $n$ 527); (d) WC in men ( $n 500)$. The bottom and top edge of the box represent the first and third quartiles (interquartile range); the line within the box represents the median; the ends of the bottom and top whiskers represent the upper and lower adjacent values; and the dots represent outliers (oBMI, objectively measured BMI; WC, waist circumference; RHINE III, Respiratory Health in Northern Europe survey)

The correlation coefficient between oBMI and figural scale rating was higher for women $(0 \cdot 77)$ than for men $(0 \cdot 70$; both $P<0 \cdot 001$, sex difference $P<0 \cdot 007)$. The differences between sBMI and oBMI were marginal, with a tendency towards under-reporting at higher figural scales and overreporting at lower figural scales (Supplemental Table 3).

The modal and median scale in both women and men was 5 , corresponding to a median oBMI of $26.6 \mathrm{~kg} / \mathrm{m}^{2}$ in women and $26.3 \mathrm{~kg} / \mathrm{m}^{2}$ in men. Based on measured WC, about half of the women $(48.6 \%$ had $\mathrm{WC}>88 \mathrm{~cm}$; range $88.05-152.25 \mathrm{~cm})$ and about two-fifths of the men $(38.4 \%$ had WC > $102 \mathrm{~cm}$; range $102 \cdot 05-145.5 \mathrm{~cm}$ ) were defined as 'at risk'. Less than $1 \%$ of participants had an oBMI of $<18.5 \mathrm{~kg} / \mathrm{m}^{2}$, so no 'underweight' category was defined. Instead, these participants were included in the 'normal weight' category (see online supplementary material 1, Supplemental Table 4).

\section{Receiver-operating characteristic curves for figural scales}

The ROC curve analyses yielded high AUC values for identifying obesity (women, $\mathrm{AUC}=0.879$; men, $\mathrm{AUC}=$ 0.863), as well as overweight (women, $\mathrm{AUC}=0 \cdot 859$; men, $\mathrm{AUC}=0 \cdot 842$; Fig. 3).
When adjusted for age, the discriminatory power of the figural scales remained consistently high (women, AUC= $0 \cdot 815$; men, AUC $=0 \cdot 784$ ). The optimal sensitivity and specificity criterion (optimal criterion) for identifying metabolic risk, correctly classifying the greatest number of 'at risk' individuals and minimising false positives, was assessed separately for obese, overweight and WC (Table 1).

Differences in predictive power and optimal criterion by age were observed for both men and women. For older participants ( $\geq 52$ years) the optimal criterion remained the same, while for younger participants of both sexes $(<52$ years) the optimal criterion to identify obesity was one scale lower; for example, for women a figural scale of 4 instead of 5 . For men, ROC curve analyses also yielded a lower optimal criterion for overweight, figural scale of 4 instead of 5 . On the other hand, no differences by age were observed for WC.

\section{Validation modelling}

Figural scales alone already explained a large part of oBMI variance (adjusted $r^{2}=0.58$ for women, 0.48 for men). Adding additional model covariates improved the adjusted $r^{2}$ substantially. The validation model showed good predictive power for BMI with an adjusted $r^{2}$ of 0.67 for women and 
(a)

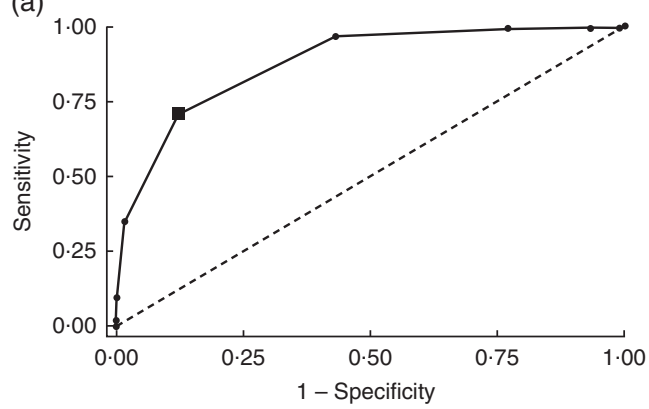

(b)

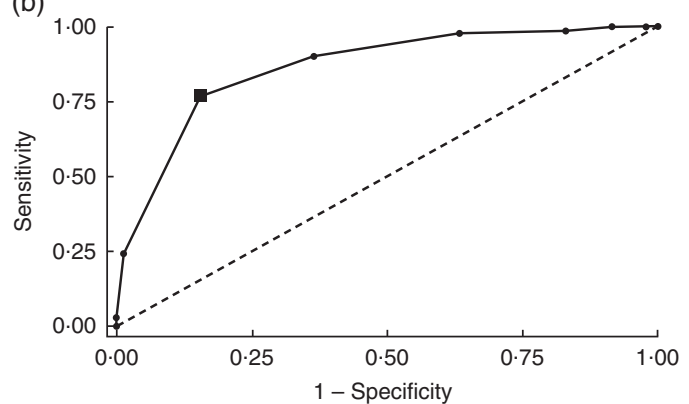

Fig. 3 ROC curves (_- data points; $\square$, cut-off point of optimal sensitivity and specificity; --- , reference line of no discrimination) for identifying obese subjects with figural scales, according to sex, in Scandinavian adults aged 38-66 years (sub-sample of the RHINE III): (a) women, sensitivity $=0.71$, specificity $=0.88, A U C=0.8786$; (b) men, sensitivity $=0.76$, specificity $=0.85, A U C=0.8630$ (ROC, receiver-operating characteristic; RHINE III, Respiratory Health in Northern Europe survey; AUC, area under the curve)

Table 1 Discriminatory capabilities of figural scales for identifying obesity, overweight and increased WC, according to sex, in Scandinavian adults aged 38-66 years (sub-sample of the RHINE III): results of ROC curve analyses

\begin{tabular}{lcccc}
\hline & AUC & $\begin{array}{c}\text { Figural scale with optimal } \\
\text { sensitivity and specificity }\end{array}$ & Youden index & Sensitivity/specificity \\
\hline Women, obese & 0.88 & 5 & 0.588 & $0.71 / 0.88$ \\
Men, obese & 0.86 & 6 & 0.611 & $0.76 / 0.85$ \\
Women, overweight & 0.86 & 4 & 0.589 & $0.80 / 0.87$ \\
Men, overweight & 0.84 & 5 & 0.513 & $0.62 / 0.89$ \\
Women, WC $>88 \mathrm{~cm}$ & 0.78 & 4 & 0.544 & $0.81 / 0.75$ \\
Men, WC $>120 \mathrm{~cm}$ & 0.81 & 5 & 0.611 & $0.83 / 0.78$ \\
\hline
\end{tabular}

WC, waist circumference; RHINE III, Respiratory Health in Northern Europe survey; AUC, area under the curve; ROC, receiveroperating characteristic.

Obese, $\mathrm{BMI} \geq 30.0 \mathrm{~kg} / \mathrm{m}^{2}$; overweight, $\mathrm{BMI} \geq 25.0 \mathrm{~kg} / \mathrm{m}^{2}$.

$0 \cdot 52$ for men. When potential influential data points, based on Cook's distance estimation, were excluded from the data set, the adjusted $r^{2}$ increased to 0.74 in women and 0.62 in men. However, the effect estimates did not change considerably. The multivariable linear regression models we obtained for predicting BMI based on figural scales were as follows.

For women:

$$
\begin{aligned}
\log \mathrm{BMI} & =2 \cdot 73661+\text { figural scales } \times(0 \cdot 0987671) \\
& + \text { age } \times(0 \cdot 0004111)+\text { educational status } \\
& \times(-0 \cdot 0169593)+\text { ever asthma } \times(0 \cdot 0246553) \\
& + \text { diabetes } \times(0 \cdot 0705768)+\text { MP symptoms ever } \\
& \times(-0 \cdot 0302591)+\text { HRT current use } \times(0 \cdot 0303381) \\
& + \text { centre } \times(-0 \cdot 0001263) .
\end{aligned}
$$

For men:

$$
\begin{aligned}
\log \mathrm{BMI} & =3 \cdot 152997+\text { figural scales } \times(0 \cdot 0564805) \\
& + \text { age } \times(-0 \cdot 0028301)+\text { educational status } \\
& \times(-0 \cdot 0071292)+\mathrm{CVD} \times(0 \cdot 0542313) \\
& + \text { smoking status } \times(-0 \cdot 0243481)+\text { centre } \\
& \times(-0 \cdot 000238)
\end{aligned}
$$

The mean difference between predicted BMI and oBMI was -0.01 (SD $2 \cdot 64) \mathrm{kg} / \mathrm{m}^{2}$ for women and -0.033 (SD 2.87) $\mathrm{kg} / \mathrm{m}^{2}$ for men. The negative values imply that the predicted measure was higher than the measured value.

\section{Discussion}

Our validation study shows that the figural scales used in RHINE III are a reliable means of identifying populations 'at risk', for both men and women. They can identify subjects 'at risk' with high accuracy, as shown by the ROC curve analyses with an AUC well above 0.8, which is of high value to studies on chronic disease development. Both sexes reported their current figural scales in accordance with objectively measured current BMI and WC. The figural scales performed well in the validation analyses, with highly consistent results across various validation approaches. The analyses could also validate BMI based on self-reported weight and height.

In general, the figural scales were well reported by RHINE participants, with a small degree of missing information in the full study (6.6\%). We observed some differences between responders and non-responders. Older, less educated and male participants were less inclined to fill in the figural scales.

Objectively measured BMI corresponded very well with the expected increase at each figural scale. Correlation 
was high between the two methods and comparable to correlations found by Bulik et al. (females, $r=0 \cdot 81$; males, $r=0.73)^{(18)}$. Some studies do not report equally high correlations, but the finding of higher correlation for women than for men is consistently observed in studies ${ }^{(21-23)}$. Correlations were also high for self-reported and objectively measured BMI, with the expected degree of over- and under-reporting depending on the figural scale.

We observed an overlap of BMI ranges across the figural scales, as expected. The ordinal and fixed scale forces people to decide on one figure or the other, even though they might feel they are in between two figural scales. This may result in a greater range of $\mathrm{BMI}^{(24)}$. The larger variability of objectively measured BMI observed at the extreme ends of the figural scales relates to the smaller number of participants found at these extremes, compared with other studies. In addition, pathological misconceptions of body weight and body image would be reflected in the extreme ends of the figural scales and may also explain some of the variation in these extreme categories.

The ROC curve analyses provided optimal sensitivity and specificity criteria for metabolic risk in this Scandinavian population, both in men and women. For younger men and women, the optimal criterion for obesity (and for overweight among men) was one figural scale lower than for older subjects. This is possibly due to more muscle mass in younger persons than in the elderly. While we cannot fully explain this observation, WC showed no age group difference, supporting our hypothesis. Younger people might also be more conscious about body norms in their age group and report lower figural scales. The optimal criteria for metabolic risk based on measured WC were the same as for overweight. The similarity between overweight and obesity criteria implies that while figural scales have a high power to identify subjects 'at risk', differentiating between those overweight and obese is more difficult. ROC curve analyses and calculated optimal criteria are similar to findings in other validation studies $^{(13,18,24)}$. The importance of calculating optimal sensitivity and specificity criteria using objective data within a study population is underlined by Madrigal et al., who compared researchers' and participants' perception of the figures and found considerable discrepancy leading ultimately to a considerable misclassification ${ }^{(22)}$. Differential interpretation of the figural stimuli has also been shown by ethnicity, with different ethnic groups assigning different BMI to the same figural stimuli ${ }^{(21,25,26)}$.

Figural scales alone explained about $50 \%$ of BMI variance, comparable to the $r^{2}$ published by KauferHorwitz et $a l^{(24)}$ and Bulik et al. ${ }^{(18)}$. The explanatory power increased when additional subjective data were added to the predictive model and after excluding significant outliers. The mean difference between predicted and objectively measured BMI was only 1 BMI unit. We observed larger, non-significant differences in the objective measures at the extreme figural scales (extremely lean, figural scale 1 and extremely obese, figural scale 9). Researchers wishing to use figural scales to estimate BMI are advised to collect these additional data, where possible, to achieve the highest degree of accuracy.

Some study limitations need to be considered when applying the figural scales. First, misconceptions of body size and weight might be differential and reporting bias, for example by sex, education and overweight ${ }^{(27)}$ or due to psychiatric disorders ${ }^{(28)}$, cannot be totally excluded. Therefore, at the individual level, there is a risk of misclassification when using figural scales. At the population level, however, the instrument discriminates well between individuals without or 'at risk'.

Second, participants were recruited randomly and should represent the general population living at the respective study sites, all set in Nordic countries. For that reason, our results are generalizable to Northern Europe or similar European countries only. We had no additional data on ethnicity, which could have introduced non-differential misclassification and loss of power ${ }^{(25)}$.

Third, the sample yields a high prevalence of asthma. There is, however, no reason to believe that asthmatics would have a differential perception of their body image. While obesity is known as a risk factor of asthma, we did not observe a high prevalence of obesity in our study population. Altogether, we do not assume differential misclassification due to the asthmatics in our study.

Finally, few participants were found at the extreme figural scales and thus there was insufficient power to calculate corresponding BMI or cut-off points for underweight subjects.

In summary, subjects 'at risk', as defined by BMI and WC, can be identified with high accuracy using this figural scale. The reliability of the RHINE III figural stimuli for a Scandinavian population is comparable to figural stimuli applied in other, partially ethnically diverse populationbased studies. Given the good performance of the figural stimuli, we will further investigate their use in public health and clinical studies. The figural scales are a valid alternative or even an additional instrument to assess anthropometric data in public health research.

\section{Supplementary material}

To view supplementary material for this article, please visit http://dx.doi.org/10.1017/S136898001600015X

\section{Acknowledgements}

Financial support: C.S., V.S., B.B. and T.G. are members of the COST Action BM1201. J.D. received a research scholarship from the COST Action BM1201 (COST-STSMECOST-STSM-BM1201-160913-036015). The RHINE III was supported financially by the Norwegian Research 
Council (grant number 214123); the Bergen Medical Research Foundation; the Western Norwegian Regional Health Authorities (grant numbers 911892 and 911 631); the Norwegian Labour Inspection; the Norwegian Asthma and Allergy Association; the Faculty of Health of Aarhus University (project number 240008); The Wood Dust Foundation (project number 444508795); the Danish Lung Association; the Swedish Heart and Lung Foundation; the Vårdal Foundation for Health Care Science and Allergy Research; the Swedish Council for Working Life and Social Research; the Bror Hjerpstedt Foundation; the Swedish Asthma and Allergy Association; the Icelandic Research Council; and the Estonian Science Foundation (grant number 4350). ECRHS acknowledgements are provided in the online supplementary material 2. Conflict of interest: The authors declare that they have no conflict of interest. Authorship: J.D., main author, developed the analytic plan presented in this paper; she conducted the analyses, interpreted the data in conjunction with all authors, and was responsible for writing the first and all consecutive drafts of the submitted manuscript. R.B. was involved in the data collection in Norway; she participated in the interpretation of results in conjunction with all authors and provided critical commentary in the draft revisions C.J. is the principal investigator (PI) of the RHINE study and is in the ECRHS study directorate; he participated in the interpretation of results in conjunction with all authors and provided critical commentary in the draft revisions. A.J. was involved in the RHINE protocol development, the data collection in Norway, the preparation of the RHINE cohort data set and provided statistical support; she participated in the interpretation of results in conjunction with all authors and provided critical commentary in the draft revisions. B.B. was involved in the data collection in Iceland; she participated in the interpretation of results in conjunction with all authors and provided critical commentary in the draft revisions. L.B. was involved in the data collection in Umeå, Sweden; he participated in the interpretation of results in conjunction with all authors and provided critical commentary in the draft revisions. B.F. is the PI of the study centre in Umeå, Sweden; he participated in the interpretation of results in conjunction with all authors and provided critical commentary in the draft revisions. T.G. is the PI of the study centre in Iceland and is in the RHINE and ECRHS study directorate; he participated in the interpretation of results in conjunction with all authors and provided critical commentary in the draft revisions. D.J. is the study coordinator of ECRHS; she participated in the interpretation of results in conjunction with all authors and provided critical commentary in the draft revisions. R.J. is the PI of the study centre in Tartu, Estonia and is a member of the RHINE study directorate; he participated in the interpretation of results in conjunction with all authors and provided critical commentary in the draft revisions. E.L. was involved in the data collection in Umeå, Sweden; she participated in the interpretation of results in conjunction with all authors and provided critical commentary in the draft revisions. D.N. was involved in the data collection in Uppsala, Sweden; he participated in the interpretation of results in conjunction with all authors and provided critical commentary in the draft revisions. E.O. is the PI of the study centre in Bergen, Norway and is a member of the RHINE study directorate; he participated in the interpretation of results in conjunction with all authors and provided critical commentary in the draft revisions. V.S is the PI in the study centre in Aarhus, Denmark and is a member of the RHINE study directorate; she participated in the interpretation of results in conjunction with all authors and provided critical commentary in the draft revisions. T.D.S. was involved in the data collection in Bergen, Norway; she participated in the interpretation of results in conjunction with all authors and provided critical commentary in the draft revisions. T.S. was involved in the data collection in Aarhus, Denmark; he participated in the interpretation of results in conjunction with all authors and provided critical commentary in the draft revisions. K.T. is the PI in Gothenburg, Sweden and is in the RHINE and ECRHS study directorate; he participated in the interpretation of results in conjunction with all authors and provided critical commentary in the draft revisions. M.W. was involved in the data collection in Bergen, Norway; she participated in the interpretation of results in conjunction with all authors and provided critical commentary in the draft revisions. G.W. was involved in the data collection in Uppsala, Sweden; she participated in the interpretation of results in conjunction with all authors and provided critical commentary in the draft revisions. S.C.D. is the PI of ECRHS, Melbourne, Australia; she participated in the interpretation of results in conjunction with all authors and provided critical commentary in the draft revisions. C.S. participated in the study protocol development and data collection in Bergen, Norway and is in the ECRHS study directorate; she participated in the development of the analytic plan, interpretation of the results in conjunction with all authors and provided critical commentary in the draft revisions. F.G.R. developed the body silhouette protocol for RHINE and ECRHS, and participated in the study protocol development and data collection in Bergen, Norway; he participated in the development of the analytic plan, interpretation of results in conjunction with all authors and provided critical commentary in the draft revisions. C.S. and F.G.R. share last authorship. Ethics of buman subject participation: The study was approved by the local ethical commissions of each study centre. All participants provided written informed consent.

\section{References}

1. Seidell JC, Kahn HS, Williamson DF et al. (2001) Report from a Centers for Disease Control and Prevention Workshop on use of adult anthropometry for public health and primary health care. Am J Clin Nutr 73, 123-126. 
2. Ali Z \& Ulrik CS (2013) Obesity and asthma: a coincidence or a causal relationship? A systematic review. Respir Med 107, 1287-1300.

3. Beuther DA, Weiss ST \& Sutherland ER (2006) Obesity and asthma. Am J Respir Crit Care Med 174, 112-119.

4. Fantuzzi G (2005) Adipose tissue, adipokines, and inflammation. J Allergy Clin Immunol 115, 911-919.

5. Canoy D, Luben R, Welch A et al. (2004) Abdominal obesity and respiratory function in men and women in the EPIC-Norfolk Study, United Kingdom. Am J Epidemiol 159, 1140-1149.

6. Leone N, Courbon D, Thomas F et al. (2009) Lung function impairment and metabolic syndrome. Am J Respir Crit Care Med 179, 509-516.

7. Rastogi D, Canfield SM, Andrade A et al. (2012) Obesityassociated asthma in children: a distinct entity. Chest 141, 895-905.

8. Jaffrin MY (2009) Body composition determination by bioimpedance: an update. Curr Opin Clin Nutr Metab Care 12, 482-486.

9. Sorensen TI \& Stunkard AJ (1993) Does obesity run in families because of genes? An adoption study using silhouettes as a measure of obesity. Acta Psychiatr Scand Suppl 370, 67-72.

10. Romieu I, Escamilla-Núñez MC, Sánchez-Zamorano LM et al. (2012) The association between body shape silhouette and dietary pattern among Mexican women. Public Health Nutr 15, 116-125.

11. Tehard B, Liere MJV, Nougué CC et al. (2002) Anthropometric measurements and body silhouette of women: validity and perception. J Am Diet Assoc 102, 1779-1784.

12. Munoz-Cachon MJ, Salces I, Arroyo M et al. (2009) Overweight and obesity: prediction by silhouettes in young adults. Obesity (Silver Spring) 17, 545-549.

13. Kabir Y, Zafar TA \& Waslien C (2013) Relationship between perceived body image and recorded body mass index among Kuwaiti female university students. Women Health 53, 693-705.

14. Leonhard ML \& Barry NJ (1998) Body image and obesity: effects of gender and weight on perceptual measures of body image. Addict Behav 23, 31-34.

15. Burney PG, Luczynska C, Chinn S et al. (1994) The European Community Respiratory Health Survey. Eur Respir J 7, 954-960.
16. Johannessen A, Verlato G, Benediktsdottir B et al. (2014) Longterm follow-up in European respiratory health studies patterns and implications. BMC Pulm Med 14, 63.

17. World Health Organization (2008) Waist Circumference and Waist-Hip Ratio: Report of a WHO Expert Consultation. Geneva: WHO, Department of Nutrition for Health and Development.

18. Bulik CM, Wade TD, Heath AC et al. (2001) Relating body mass index to figural stimuli: population-based normative data for Caucasians. Int $J$ Obes Relat Metab Disord 25, 1517-1524.

19. Fawcett T (2006) An introduction to ROC analysis. Pattern Recogn Lett 27, 861-874.

20. Burnham KP \& Anderson DR (2004) Multimodel inference understanding AIC and BIC in model selection. Sociol Method Res 33, 261-304.

21. Fitzgibbon ML, Blackman LR \& Avellone ME (2000) The relationship between body image discrepancy and body mass index across ethnic groups. Obes Res 8, 582-589.

22. Madrigal H, Sanchez-Villegas A, Martinez-Gonzalez MA et al. (2000) Underestimation of body mass index through perceived body image as compared to self-reported body mass index in the European Union. Public Health 114, 468-473.

23. Martinez JA, Kearney JM, Kafatos A et al. (1999) Variables independently associated with self-reported obesity in the European Union. Public Health Nutr 2, 125-133.

24. Kaufer-Horwitz M, Martinez J, Goti-Rodriguez LM et al. (2006) Association between measured BMI and self-perceived body size in Mexican adults. Ann Hum Biol 33, 536-545.

25. Kronenfeld LW, Reba-Harrelson L, Von Holle A et al. (2010) Ethnic and racial differences in body size perception and satisfaction. Body Image 7, 131-136.

26. Mciza Z, Goedecke JH, Steyn NP et al. (2005) Development and validation of instruments measuring body image and body weight dissatisfaction in South African mothers and their daughters. Public Health Nutr 8, 509-519.

27. Paeratakul S, White MA, Williamson DA et al. (2002) Sex, race/ethnicity, socioeconomic status, and BMI in relation to self-perception of overweight. Obes Res 10, 345-350.

28. Torres-McGehee TM, Monsma EV, Dompier TP et al. (2012) Eating disorder risk and the role of clothing in collegiate cheerleaders' body images. J Athl Train 47, 541-548. 